\title{
Hybrid aortic arch repair: The ultimate solution or a stop along the way to a total endovascular arch reconstruction?
}

\author{
George J. Arnaoutakis, MD, and Wilson Y. Szeto, MD
}

\author{
From the Division of Cardiovascular Surgery, University of Pennsylvania, Philadelphia, Pa. \\ Disclosures: W.Y.S. is a consultant and member of an advisory board for Micro Interventional Devices, Inc, and \\ has research grants from Edwards Lifesciences Corporation, Medtronic, Inc, LivaNova, and Bolton Medical. \\ The other author has nothing to disclose with regard to commercial support. \\ Received for publication March 30, 2016; accepted for publication March 31, 2016. \\ Address for reprints: Wilson Y. Szeto, MD, Division of Cardiovascular Surgery, Penn Presbyterian Medical \\ Center, University of Pennsylvania, 51 N 39th St, Heart and Vascular Pavilion 2A, Philadelphia, PA 19104 \\ (E-mail: Wilson.szeto@uphs.upenn.edu). \\ J Thorac Cardiovasc Surg 2016;152:169-70 \\ $0022-5223 / \$ 36.00$ \\ Copyright (c) 2016 by The American Association for Thoracic Surgery \\ http://dx.doi.org/10.1016/j.jtcvs.2016.03.064
}

In their article in this issue of The Journal, Faure and colleagues $^{1}$ report their single-center experience with hybrid repair of chronic dissection-related aortic arch aneurysms. They combined aortic great vessel debranching with thoracic endovascular aortic repair to address the aortic arch pathology completely. In this series of 33 patients, there were no in-hospital deaths. At a respectable mean follow-up of 20 months, the overall mortality was $12 \%$ at last follow-up. There was a $24 \%$ incidence of endoleak overall, and 8 patients required some form of major reintervention, not all of which were for endoleak. Given this high-risk group of patients, Faure and colleagues ${ }^{1}$ are to be commended on their excellent outcomes.

Surgical treatments to address aortic arch pathology traditionally have involved open techniques of total arch replacement with circulatory arrest and reimplantation of the great arteries. Even in a high-risk patient population, excellent results can be achieved, and many centers continue to advocate this open surgical approach as the criterion standard. ${ }^{2}$ The rapid expansion of thoracic endovascular technology in the past 15 years, however, is transforming traditional open repair paradigms. Furthermore, innovative hybrid repair approaches, such as those described by Faure and colleagues, ${ }^{1}$ with a combination of open and endovascular techniques, have expanded the risk profile of patients and scope of aortic pathologies amenable to surgical therapies. ${ }^{3-6}$ With continued evolution in this field, we need to reassess critically the current status of surgical options for treating aortic arch pathologies. It is also imperative that we seek collaborative approaches to studying aortic arch aneurysm in the setting of chronic dissection.

To devise the optimal treatment strategy for arch pathology, it is important to consider the aortic arch together with any pathology of the ascending or descending thoracic aorta. Furthermore, the acuity as well as the burden of comorbidities must be carefully assessed. These factors

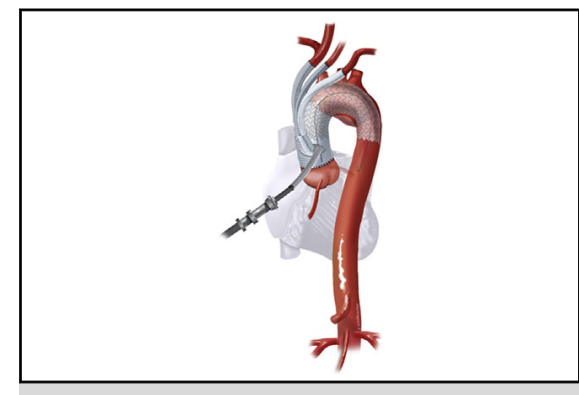

Type II hybrid arch with ascending aorta replacement and zone 0 endograft deployment.

\section{Central Message}

Hybrid arch repair combines open aortic great vessel debranching with thoracic endovascular technology to address various arch pathologies without requiring prolonged periods of circulatory arrest.

See Article page 162.

not only dictate the extent of required aortic reconstruction at the index operation but also affect whether a given patient can tolerate an extensive single-stage operation. In patients with a heavy burden of comorbidities, a multistage therapeutic strategy may be best.

It is important to note that during the study period Faure and colleagues ${ }^{1}$ undertook only hybrid repairs for chronic dissection-related arch aneurysms. Although at present many aortic centers would consider traditional open repair the criterion standard for chronic arch aneurysm, Faure and colleagues ${ }^{1}$ seem to have shifted their treatment paradigm to offering the hybrid approach routinely. Despite the well-studied durability of the open approach, the morbidity and mortality associated with open repair remain significant. It would have been interesting if there had been a contemporaneous group of patients who underwent traditional open repair for comparison; however, the outcomes reported in this study shed light on the early and midterm complications that may arise from hybrid arch repair, particularly the occurrence of endoleak (both early and delayed) and need for repeat reinterventions. The need for aortic reintervention occurred in nearly a quarter of patients, which is consistent with other reports and highlights the need for ongoing surveillance in patients undergoing this treatment strategy.

The study of Faure and colleagues ${ }^{1}$ is strengthened by its reporting of aortic remodeling measurements, and it is 


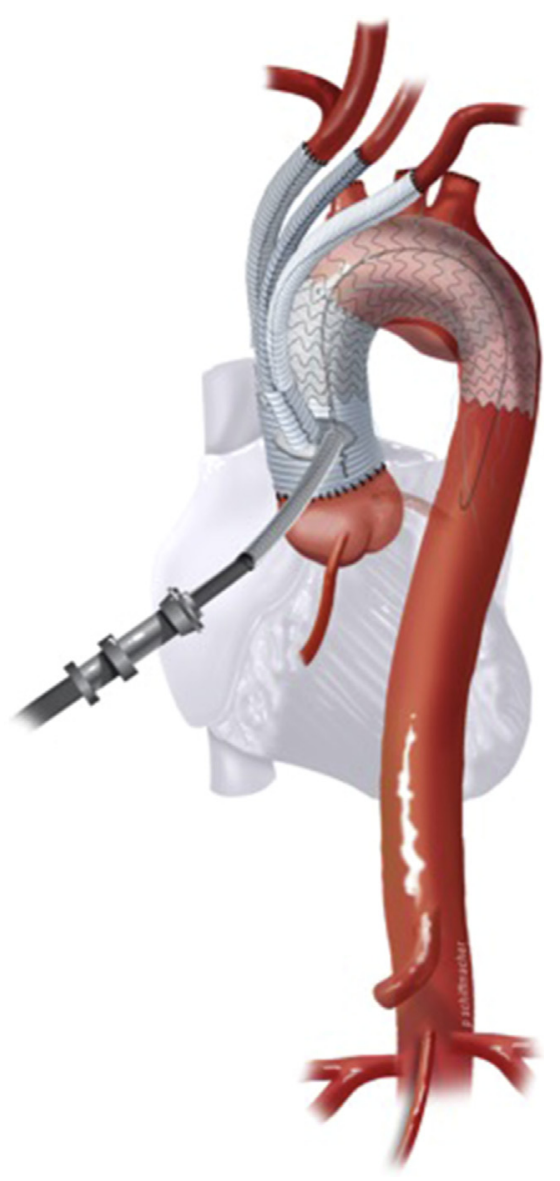

FIGURE 1. Type II hybrid arch with ascending aorta replacement and zone 0 endograft deployment.

important that future studies consider late aortic remodeling figures as well. Faure and colleagues ${ }^{1}$ observed complete false-lumen thrombosis in $76 \%$ of the cohort; other measurements of aortic remodeling were relatively favorable as well. When applied in the dissected aorta, however, the long-term effects of stent-graft therapy on late aortic remodeling remain unknown. More data are needed before commentary can be made comparing the durability of the endovascular approach versus traditional open repair.

We have adopted the hybrid approach in many instances (Figure 1). ${ }^{3}$ Until more advanced stent-graft technology is available that specifically addresses the aortic arch, we believe that the hybrid approach complements rather than competes with traditional open techniques. Many patients who would not be candidates for traditional open repair because of their comorbid burdens are candidates for the hybrid approach.

Complicated arch pathology that encompasses both the proximal ascending and the distal descending aorta remains a clinical challenge. It is incumbent on cardiovascular surgeons to welcome innovation and be early adopters of these new treatment paradigms for aortic arch pathology. Technologic advancements should not be viewed as obstacles but rather as motivation to enhance surgical techniques and improve outcomes. We must maintain equipoise, however, tempering our enthusiasm for new therapies with our responsibility to scrutinize these new approaches critically and to conduct evidence-based investigations. Long-term follow-up and prospective randomized data are necessary before the true impact of these new hybrid strategies will be fully understood. With this balanced approach, we can usher in the endovascular era for aortic arch pathology in a controlled manner and simultaneously offer therapeutic interventions for patients who had no surgical options in the past. Faure and colleagues ${ }^{1}$ are to be congratulated for their important contribution to this field.

\section{References}

1. Faure EM, Canaud L, Marty-Ané C, Alric P. Hybrid aortic arch repair for dissecting aneurysm. J Thorac Cardiovasc Surg. 2016;152:162-8.

2. Iafrancesco M, Ranasinghe AM, Dronavalli V, Adam DJ, Claridge MW, Riley P, et al. Open aortic arch replacement in high-risk patients: the gold standard $\dagger$. Eur J Cardiothorac Surg. 2016;49:646-51.

3. Bavaria J, Vallabhajosyula P, Moeller P, Szeto W, Desai N, Pochettino A. Hybrid approaches in the treatment of aortic arch aneurysms: Postoperative and midterm outcomes. J Thorac Cardiovasc Surg. 2013;145(3 Suppl): S85-90.

4. Cao P, De Rango P, Czerny M, Evangelista A, Fattori R, Nienaber C, et al. Systematic review of clinical outcomes in hybrid procedures for aortic arch dissections and other arch diseases. J Thorac Cardiovasc Surg. 2012;144: 1286-300. 1300.e1-2.

5. Preventza O, Garcia A, Cooley DA, Haywood-Watson RJ, Simpson K, Bakaeen FG, et al. Total aortic arch replacement: a comparative study of zone 0 hybrid arch exclusion versus traditional open repair. J Thorac Cardiovasc Surg. 2015;150:1591-8; discussion 1598-600.

6. Vallabhajosyula P, Szeto WY. Current paradigms in aortic arch repair: striking the balance between open surgery and endovascular repair. J Thorac Cardiovasc Surg. 2015;150:1399-400. 